Investigación

\title{
EL CONCEPTO CULTURAL DE BIENESTAR EN NIÑAS Y NIÑOS MAPUCHE: PERSPECTIVAS DE SUS CUIDADORES Y AUTORIDADES ANCESTRALES
}

THE WELL-BEING CONCEPT OF MAPUCHE CHILDREN: CULTURAL PERSPECTIVES OF THEIR CAREGIVERS AND ANCESTRAL AUTHORITIES

\section{Ana María Alarcón Muñoz}

Enfermera, Antropóloga. PhD Antropología

Depto. Salud Pública, Universidad de la Frontera, Temuco, Chile

ana.alarcon@ufrontera.cl

https://orcid.org/0000-0003-0706-478X

\section{Raúl Enrique Garcés Zúñiga}

Enfermero. Magister Salud Pública

Universidad de la Frontera, Temuco, Chile

r.garceszuniga@gmail.com

https://orcid.org/0000-0001-7774-2588

\section{Yolanda Nahuelcheo Saldaña}

Trabajadora Social

Depto. Salud Pública, Universidad de la Frontera, Temuco, Chile

yoalnasa@gmail.com

https://orcid.org/0000-0002-3010-0470

\section{Marcela Castro Garrido}

Antropóloga. Magister Ciencias Sociales y Género

Depto. Salud Pública, Universidad de la Frontera, Temuco, Chile

Marcela.castro@ufrontera.cl

https://orcid.org/0000-0003-0404-7503

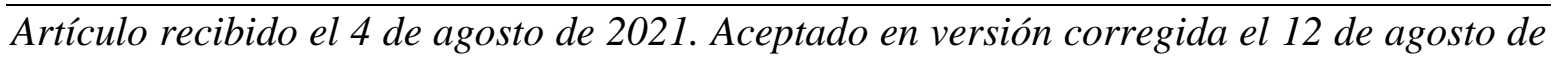
2021.

\section{RESUMEN}

El concepto de salud y bienestar es cultural, social e históricamente dependiente, y al mismo tiempo obedece a diversos enfoques socio-políticos. Lo que se considera sano, enfermo, o en buenas condiciones generales de salud depende de los valores y expectativas que se tiene de las personas y sus cuerpos en las diversas culturas. En nuestro país la infancia mapuche se encuentra en una interfaz entre valores de la modernidad y aquellos propias del mundo mapuche; así sus cuidadores velan por la protección y traspaso de valores culturales esenciales. Este estudio, se propone indagar en los conceptos de salud, bienestar y 
enfermedad asociados al desarrollo y crianza de niños y niñas mapuche desde la perspectiva de sus cuidadores, familiares y autoridades ancestrales. Es un diseño cualitativo basado en Teoría Fundamentada; los datos se recolectaron mediante entrevistas en profundidad y observaciones dirigidas hacia el cuidado infantil en el propio ambiente familiar. El análisis se realizó en el programa Atlas ti.8, mediante segmentación, codificación y elaboración de categorías. Los resultados indican que salud y bienestar se traduce en el concepto küme mongen, küme felen (estar bien, en armonía con todo). Así, estar sano es crecer conforme al entorno, como una planta que se alimenta naturalmente, los niños/as tienen ganas de nutrirse, no tienen enfermedades y sus cuerpos son firmes y fuertes. Niños y niñas deben demostrar la adquisición de destrezas especialmente autonomía, caerse y levantarse sin llorar, no ser temerosos, ser activos, atentos a mirar y escuchar lo que ocurre a su alrededor e interesados en aprender.

Palabras clave: Salud, bienestar, infancia mapuche.

\section{ABSTRACT}

The concept of health and well-being is culturally, socially and historically dependent, and at the same time is dependent of various socio-political approaches. What is considered healthy or sick, depends on the values and expectations that people have in different cultures. In our country, Mapuche childhood is at in the interface between the values of modernity and those of the Mapuche world. Thus, their caregivers ensure the protection and transfer of essential cultural values. This study aims to investigate the concepts of children health and well-being, from their caregivers, relatives, and ancestral authorities' perspectives. It is a qualitative design based on Grounded Theory; the data were collected through in-depth interviews and observations. The analysis was carried out in the Atlas ti.8 program, through segmentation, coding and categories construction. The results indicate that health and wellbeing correspond to the mapuche concept of küme mongen, küme felen (means to be well, being in harmony with everything). Thus, to be healthy is to grow according to the environment and spiritual context, like a plant that feeds naturally children want to nourish themselves, their bodies must be firm and strong. Boys and girls must demonstrate the acquisition of certain skills especially autonomy, for example, to be strong, active and attentive to look and listen to what is happening around them and interested in learning.

Keywords: Health, well-being, mapuche childhood.

\section{http://dx.doi.org/10.7764/Horiz_Enferm.32.2.140-154}

\section{INTRODUCCIÓN}

La salud y el bienestar en los seres humanos son conceptos amplios que varían de acuerdo con el contexto cultural, social, político y también histórico. De manera transversal, ambos conceptos son fundamentales para el desarrollo pleno de las personas en el entorno en que se desenvuelven. La salud como concepto 
Alarcón Muñoz AM, Garcés Zúñiga RE, Nahuelcheo Saldaña Y, Castro Garrido M

tiene múltiples acepciones las que dependen de los enfoques en uso y la necesidad de dar respuesta a diversas interrogantes y análisis socio-políticos necesarios $^{(1)}$.

La infancia, por otro lado, es un periodo vital del desarrollo humano que constituye la clave para el futuro de la sociedad. Su importancia y bienestar implica analizar y discutir elementos multifactoriales de orden sistémico, político, sociales y culturales, que trascenderán para el futuro de la sociedad $^{(2)}$.

Así, salud e infancia constituyen un fenómeno socio-cultural y político que puede revestir importantes desafíos para la investigación y la política social de un país $^{(3)}$. La crianza y cuidado de la infancia indígena es un proceso compartido de participación y enseñanza de varias generaciones que con diversos roles $y$ prácticas cuidan, enseñan y refuerzan la cultura propia, tanto en áreas urbanas como rurales ${ }^{(4)}$.

En Chile, la infancia mapuche, especialmente aquella que habita en comunas rurales del sur del país, se desarrolla en un contexto sociopolítico de conflicto interétnico y desigualdad, que sitúa a los infantes mapuche en una condición de desventaja respecto a sus pares no indígena ${ }^{(5)}$. Diversos estudios han demostrado cómo la salud de los infantes se ve afectada en tiempos de conflictos bélicos, ocupación o inestabilidad social y territorial $\left({ }^{6,7,8)}\right.$.

La $\operatorname{OPS}^{(9)}$ ha señalado que la población de niños y niñas indígenas constituye una de las poblaciones más vulnerables y marginadas a nivel mundial, para la cual es urgente generar medidas político-sociales a nivel de países, que aseguren su supervivencia y calidad de vida. Diversos estudios latinoamericanos indican que desde el punto de vista sanitario los niños indígenas presentan mayores tasas de mortalidad, problemas asociados a mal nutrición y dificultades en su desarrollo general ${ }^{(10,11)}$.

El Estado de Chile reconoce nueve pueblos indígenas, siendo el mapuche el más numeroso $^{(12)}$. El 8,7\% de la población del país menor de 18 años es indígena y el 26,5\% de ella se encuentra bajo la línea de la pobreza. En la región de La Araucanía, el 35\% de los niños, niñas y adolescentes es indígena y pertenece al pueblo mapuche; el 38,4\% de ellos se encuentra bajo de la línea de la pobreza ${ }^{(13)}$. Muchos niños y niñas mapuche sufren discriminación y violencia en distintos ámbitos de sus vidas y de la sociedad en general, situación que ha sido descrita en el informe sobre violencia institucional hacia la niñez mapuche en Chile ${ }^{(5)}$.

Un informe elaborado por el Banco Mundial $^{(14)}$ indica que los niños indígenas menores de 5 años tienen 2,6 veces más riesgo de morir que los niños no indígenas, y que finalmente no logran los estándares de crecimiento y desarrollo esperados.

El sistema de salud chileno realiza un importante proceso de seguimiento del desarrollo y crecimiento infantil a través del Programa Nacional de Salud de la Infancia. En éste, la disciplina de enfermería ha tenido históricamente un rol de vigilancia y cuidado de la niñez en su entorno familiar, apoyando su salud, crecimiento y desarrollo. La enfermería que se desarrolla con poblaciones culturales $o$ en territorios indígenas, requiere tanto de conocimientos como de 
prácticas interculturales que no son iguales en contextos urbanos, no indígenas y no migrantes ${ }^{(15,16)}$. Esta enfermería reconoce la cultura del usuario, la asume como una herramienta más al servicio del cuidado de salud, su práctica se distingue por incorporar los conceptos propios de la población usuaria y lograr acuerdos en una relación de ayuda con significado ${ }^{(17)}$.

La literatura ha definido también la necesidad de observar otras formas de ser un /a niño/a, otras formas de educar y concebir la infancia, y en que la salud incorpora el sentido de la espiritualidad, importancia de la naturaleza, la familia, la comunidad, como aspectos centrales de todo ser humano ${ }^{(18,19,20)}$.

Estudios sobre la salud y cultura mapuche ${ }^{(21,22,23)}$, señalan que en la base del desarrollo de una persona -che; se encuentra, por un lado, un estado de equilibrio, salud y bienestar, y por otro un conjunto de valores culturales que rigen la conducta de las personas en sociedad. En consecuencia, el estado de bienestar -o salud-, es inseparable de los valores que conducen el crecimiento y desarrollo de los niños y niñas mapuche ${ }^{(24)}$.

En el mundo mapuche la enfermedad o kutran, puede ser el resultado de un desequilibrio en la interacción entre la persona y su ambiente natural-espiritual $^{(24) .}$ La enfermedad se opone al estado de bienestar colectivo y surge por una serie de razones que ponen en peligro el equilibrio de la familia y la comunidad. Por ejemplo, explotación indiscriminada de la naturaleza, inequidades sociales, ambiente político inseguro, todas causas que afectan al colectivo y no solo a la persona como individuo.
En general, el enfoque biomédico con el que se valora el desarrollo y salud de la infancia, no incorpora aspectos interculturales ni menos colectivos para actuar en el proceso de salud, vigilancia y protección de su desarrollo ${ }^{(25,26)}$.

En consecuencia, para la Enfermería como disciplina del cuidado, y particularmente aquella familiar y comunitaria de la infancia, es imprescindible conocer la cultura de los usuarios, los estilos de crianza, la parentalidad, los modelos educativos culturales, los valores y conceptos de salud y bienestar; de este modo llegar a entregar una atención con sentido de pertinencia y grata para la población.

Este estudio apunta a conocer los conceptos de salud y bienestar asociados a la infancia mapuche, desde la perspectiva de sus cuidadores y autoridades ancestrales. Con ello se pretende proporcionar información actualizada que contribuya a la promoción del desarrollo integral y pleno de los niños y niñas mapuche, y a mejorar la pertinencia de las acciones de enfermería en el campo de la infancia, la familia y comunidad.

\section{MATERIALES Y MÉTODOS}

Se trata de un estudio cualitativo basado en la teoría fundamentada ${ }^{(27)}$, la cual ha sido considerada como un método comparativo, flexible, y sistemático para construir teoría a partir de datos sociales, culturales y procesos psicológicos ${ }^{(28)}$. Los participantes fueron 12 cuidadores de niñas y niños mapuche de 0 a 4 años y 6 autoridades ancestrales, todos habitantes de cuatro localidades de la región de La Araucanía, con la más alta proporción de población mapuche y ubicadas en diversas 
Alarcón Muñoz AM, Garcés Zúñiga RE, Nahuelcheo Saldaña Y, Castro Garrido M

entidades geo-territoriales (cordillera, valle y costa). El acceso a las familias fue a través de asesores culturales y miembros de organizaciones de las localidades seleccionadas, quienes acompañaron al equipo de investigadores en la elección y visita a los participantes. De acuerdo a los asesores, los seleccionados contaban con el criterio de ejemplaridad de una familia mapuche, es decir coexistían al menos dos generaciones en un mismo espacio, hablaban el mapudungun y estaban al cuidado de niños pequeños. En total los participantes fueron 11 mujeres y 7 hombres y sus edades fluctuaron entre los 19 y 92 años; eran madres, padres, abuelos/as, machi hombre y mujer, asesores culturales y una profesora intercultural. Los datos se recolectaron mediante entrevistas en profundidad basadas en una pauta orientada por los objetivos del estudio y observaciones dirigidas hacia la interacción de adultos con niños/as; se realizaron varias visitas por familia hasta saturar la información obtenida'. Ello implicó aproximadamente un año de trabajo de campo con acompañamiento semanal a las familias del estudio. Los datos se registraron en un cuaderno de campo, algunas observaciones fueron video-grabadas y las conversaciones fueron audio-grabadas. Cada miembro de la familia firmó un consentimiento informado el cual había sido aprobado por el comité de ética científico de la Universidad de La Frontera y del Servicio de Salud Araucanía Sur.

Los registros transcritos en el software "F5 transcription de IOS", fueron ingresados en el programa Atlas.ti8 para su análisis. Este fue realizado por dos miembros del equipo y consistió en: segmentar los datos en unidades de significados mediante una lectura constante de los registros, realizar codificación teórica y en-vivo de cada segmento seleccionado, analizar los códigos de acuerdo a similitudes y diferencias y agruparlos, generar unidades de significados similares con los códigos denominándoles categorías, y configurar una interpretación de cada categoría para generar la teoría emergente a partir de los datos. Cada una de las categorías se apoya en la evidencia cualitativa constituida por las propias citas de los participantes. El rigor y fiabilidad de la investigación está cubierta por el registro de cada una de las actividades monitoreada por el comité de ética, y por la presencia de múltiples métodos para obtener información; así como también un análisis realizado por dos investigadoras quienes discutían los hallazgos y seleccionaban los párrafos ejemplificadores $^{(29)}$.

\section{RESULTADOS}

Los resultados se ordenan primero, señalando el contexto en el que se desarrolla la vida de los y las niñas mapuche en una comunidad rural. En segundo lugar, se describe el concepto general de salud y bienestar desde la cultura mapuche, y finalmente los aspectos específicos asociados a la salud de la infancia mapuche como, por ejemplo: crecimiento, alimentación, destrezas, habla-socialización con sus pares y adultos en el entorno comunitario y finalmente la importancia del cariño y alegría como una señal de salud. 


\section{Rukawe (lugar en donde se emplaza la vivienda y familia mapuche)}

Todas las familias que participaban en el estudio vivían en sectores rurales. Al acercarse al sitio de la vivienda familiar se podía observar la presencia cercana de varias viviendas de madera con pequeños caminos de conexión entre ellas. Estas correspondían en general a la casa de los abuelos, de tíos y primos y de la familia índice del estudio. Había un espacio de huerta, de crianza de animales pequeños y de extracción de agua. Niños y niñas iban de casa en casa, se quedaban a almorzar en una y luego jugaban entre todos, o también podían volver a su casa con sus cuidadores principales; muchas veces llevaban encargos de un lugar a otro como una forma en que la familia compartía alimentos o productos. Los adultos también comparten en algún momento del día para tomar mate, ver una teleserie o las noticias juntos. Al caer la noche cada "microunidad familiar", vuelve a su hogar y descansa. Así, la vida de los/as niños/as pasa entre personas o parientes adultos que cuidan de ellos, como también lo hacen los niños mayores de la familia que vigilan a los más pequeños.

El contexto familiar es colectivo, con una dinámica que implica compartir productos, actividades cotidianas, relatos, conversaciones de las cuales fuimos testigos. Así adultos y niños comparten diariamente, se ayudan, se colaboran y conversan. En esas conversaciones los adultos van mostrando sus ideas de lo que es correcto, van indicando las tareas y van demostrando cómo hacer las cosas. Así, niños y niñas van aprendiendo sus roles y formas de hacer bien las cosas a través de las actividades que realizan al interior de su familia.

"Mi cuñada sabía que ustedes venían y trajo una lasaña para compartir, mi suegra hizo las sopaipillas..., ya van a venir a tomar mate cuando terminemos con su trabajo. Me dijeron... pa' que le diga a [el niño], que le vaya a avisar a la abuela y a la tía...que vienen visitas pa' que vengan a tomar mate" (registro de campo).

\section{Salud y bienestar como concepto integral}

Todos los entrevistados señalaron un concepto de salud relacionado con la idea de estar bien "kume felen", estar bien con todo "kume mongen". También significa equilibrio entre todos los elementos que conforman la naturaleza el "itrofill mongen", esto fue descrito como el entorno natural de cada comunidad, como, por ejemplo, pequeños bosques, fuentes de agua, ríos, mar o montañas. Por lo tanto, salud es un concepto que implica equilibrio entre todos los elementos del universo. La persona es una parte de ese universo y se conecta con todo lo que existe a su alrededor, -tierra, aire, fuego, hielo, masa. Así, todo lo que ocurre en el entorno afecta a la persona y su salud.

Otro aspecto de la salud y bienestar de la infancia se relaciona con los valores propios de la cultura mapuche. Muchos cuidadores señalaron que los/as niños/as sanos son también respetuosos, cariñosos, saben cómo comportarse, son generosos y correctos en todo sentido. Cuando alguna de esas características no está presente, se dice que "algo anda mal con ese chiquito... hay que corregirlo, no está bien enseñado como mapuche a o tiene algo" 
(Madre). A modo de ejemplo un padre indicó: "siempre la familia se preocupa de eso, de ir inculcándole ciertos valores, por ejemplo, cuando uno escucha de repente a un kimche (persona sabia o conocedora de la cultura) que dice que el niño tiene que ser obediente, tiene que ser buena gente, ser sabio, ser fuerte y ser como tener mucha energía, tiene que ver con los valores de lo mapuche" (Padre). "Tener un hijo es poder mantener un linaje familiar, un hijo para una persona mapuche es en quien tu puedes confiar, preparar y entregarle el conocimiento para que algún día ellos sean gente que cumplan las cuatro estructuras que los mapuches debiésemos ser; ser kümeche (bondadoso), un norche (correcto), un newenche (fuerte física y espiritualmente) y kimche (sabe de dónde viene)" (Abuelo).

Es en ese contexto, -equilibrio y valores-, en el que se aprecia la salud y bienestar de niños y niñas mapuche.

\section{Características de una infancia sana}

3.1. Que vayan creciendo normalmente según su familia

Todos los participantes mencionaron que un niño o niña sana, se nota porque va creciendo normalmente, de acuerdo a las características de su familia y las propias del/a niño/a. Así mencionan que no todos los niños son iguales en su desarrollo, lo importante que ese crecimiento sea armónico y activo. A modo de ejemplo se presentan algunas citas: "va creciendo, se nota como va estirándose más, empieza a mover, ya grita, empieza a chuparse los dedos, cositas así que hacen cuando van creciendo normal" (Madre); "va creciendo, por ejemplo, físicamente, desarrollando su cuerpo bien, yo encuentro que va bien él, es un poco desordenado, pero yo encuentro que está bien, se nota en su físico y en su actividad"(Padre), "lo que pasa es que nosotros, el sistema público o la salud ha hecho estudios que va hablando del crecimiento, el desarrollo del niño pero igual hay hartas cosas que no cuadran, ya poh, no le pueden decir que no crece si los papas son chicos, los abuelos también, está mal eso... cada niño es diferente"(Madre).

\subsection{Bien alimentados}

Se señala que los niños sanos se alimentan bien, comenzando por la lactancia materna prolongada, reciben su alimentación cocinada, natural, fresca no envasada, y también aceptan la alimentación variada que comienza a darse desde muy pequeños. Se presentan algunos extractos de conversaciones para ejemplificar este punto: "darle pecho, mucha teta para que mame bien", "el amamantamiento no tiene tiempo, hay que hacerlo hasta que los niños quieran", "mientras más pecho, más sana es la guagua y será sano también más adelante". Respecto del tipo de alimentos se mencionó: " la comida no es solamente el comer sino el alimentarse como mapuche, en la alimentación, la forma de comer, la forma de preparar la comida"; "cocinarles uno con cosas naturales, comida de huinca envasada no, comida mapuche, pankutras, una cazuela de locro"; "trato de darle comida que le alimente, que la nutra mejor"; "el mapuche pueblino come de todo, lo que le den allá pura chatarra, en cambio en el campo uno trata de evitar eso y trata de darle comida que le alimente y que le nutra mejor, que no le haga mal". La alimentación es variada desde muy chiquitos, incluso a lactantes se les da a 
probar algunos alimentos como una forma de reconocer sabores y evitar que más adelante desprecien algunos alimentos: "hay que darle agüita... darle a probar alimento saludable, si todo no les gusta es porque no les dieron cuando guagüitas", "dándoles agüitas de hierbas, sopita de legumbres para criarlos sanos", "come todo lo que yo preparo, que es la comida nuestra, todo come mi nietecita".

\subsection{Activos física e intelectualmente \\ El buen estado de salud se} demuestra a través de la actividad (juego especialmente), la vivacidad, y la atención. Muchos cuidadores señalaron que un niño despierto, atento a lo que pasa a su alrededor es una señal que se encuentra bien de salud. En ese contexto se valora mucho la vida rural, la cual le otorgaría un entorno con múltiples estímulos para su buen crecimiento. La vida natural y social proporciona los elementos para estar atentos a estímulos en forma constante. El hecho de vivir en una familia extendida, en medio de primos, hermanos, con animales, plantas, flores, y arboles; contribuye según los participantes a la salud y bienestar de los niños. A modo de ejemplo las siguientes citas: "les hace bien... estar al aire libre: salir al campo, pasear, sin timidez, salir a ver la naturaleza. Andar como corderitos saltando, ensuciarse en el barro, sentirse libre, subir y bajar árboles, respirar aire libre, sacarlos a que lo miren todo" (Agente); "bien activo...yo le hice un güiño a él, y cuando empezó a caminar y todo, tomó el güiño y empezó a jugar con su güiño, se afirmaba, caminaba con su güiño igual" (Padre); "tira a caminar...aprende ya a tomarse de algo y ya hace empeño a levantarse y ahí ya hay que tener cuidado en eso. Lo dejas sentadito, el niño ya tira sus manitos a gatear, ahí tiene a sus hermanitos que le ayudan"(Madre); "darse cuenta de todo lo que pasa alrededor, mirar, después se da cuenta del espacio y de la familia... jugar a todo lo que nos permite la naturaleza, desde pan de barro hasta meterse al monte sin dificultad, convivir con el medio, subirse a los árboles"(Padre); "de chiquitos estar mirando todo, atentos, yo lo llevo en el coche a todas partes, así me acompaña y pone atención a todo con sus ojitos" (Madre), "poder manipular todo lo que estaba cercano, nada estaba prohibido, eso , eso era una cosa que ahora no va con su nietos, otras generaciones no le permiten cruzar un cerco por ejemplo de alambre y antes nosotros éramos como culebritas" (Abuela).

\subsection{Tratan de hablar}

Otro elemento considerado un indicador de salud y bienestar en los niños es que comiencen a enunciar palabras, traten de imitar sonidos y logren comprender lo que se le dice. Por ejemplo, "los niños tienen que tratar de hablar... para uno es una alegría cuando escucha que dicen algo... una trata de ayudarle cantando, hablándole, poniéndoles música mapuche" (Madre); "entienden lo que uno le dice, desde chico entendiendo, hay que buscarle conversa como se dice... así aprende... si poh tiene que hablar... hay que ayudarle a hablar hablándole (Padre); "tiene que hablar clarito, de guagua hay que hablarle harto, inventarle los propios cuentos... dicen cositas así de simple... así empezó a hablar" (Madre). "Tienen que nombrar las cosas tal como son, bien inteligente, que escuche así va 
Alarcón Muñoz AM, Garcés Zúñiga RE, Nahuelcheo Saldaña Y, Castro Garrido M

aprendiendo la sabiduría" (Madre). Ve los animales afuera, las vacas, los pollos, los chanchos, yo le digo "mira el chancho, ¿cómo hace el chancho"? y él ya empieza "ya, mchmch" y como que lo espanta, así comienza a hablar está acostumbrado porque lo escucha siempre" (Abuela).

\subsection{Se ven alegres por el cariño que reciben}

Todos los entrevistados señalaron que un niño sano es un niño contento o alegre. Ello se interrelaciona con el valor de una persona que hace el bien. Para que esto se desarrolle, los niños deben estar en el seno de una familia que le proporcione cariño. Se presentan algunos extractos relacionados con este tema: "una familia cariñosa que permitía desarrollarse en todos los planos, artísticos, culturales, de socialización con el medio, es lo básico para ser un niño feliz... sanito" (Asesor); "el afecto es muy lindo, muy lindo, sobre todo los hermanos con sus hermanas mujeres, entonces siempre era como de protección, de vamos para allá, el que te toma la mano, y te dice te llevo, y ella se siente segura y feliz" (Padre); "la llegada del niño en la familia mapuche, es una alegría, lo fundamental de criar a un hijo es el amor, la comprensión, así ellos también entregan amor... un niño tranquilo amoroso es un niño sano"(Abuelo); "tener una guagua era tener la felicidad más grande porque de ver un hijo nacer es porque Dios lo ha enviado, son la bendición del hogar, es bonito tener hijos, enseñarles cosas bonitas, lo que uno ha aprendido por ejemplo para que estén contentos y sean felices" (Madre).

\section{La autonomía como parte de la salud y bienestar infantil}

Muchos de los relatos señalaron que una faceta del bienestar infantil es la autonomía. Para la cultura mapuche el ser fuerte es un valor importante, lo cual se demuestra como un precursor de la capacidad para hacer cosas por si mismos/as, es decir autonomía. Los relatos muestran que niños $y$ niñas deben demostrar esta autonomía de diversas maneras: "cuidar a los más pequeños siempre que estén a su alcance" (Madre); "un niño que conoce la cultura, su espacio y su entorno, es un niño capacitado, un niño muy bien desarrollado" (Asesora); "ya comienzan a hacer cosas solos, claro, antes acompañan en las tareas, pero después ya hacen sus cositas solos" (Abuelo). "Qué me gusta de eso? Que más adelante son autónomos... bien independientes... lo que pasa es que la sociedad chilena no es muy proclive a la autonomía... más bien está pensada como: haz lo que puedas" (Padre). "Es entretenido porque tienen todo, juegan, se entretienen con todo, tienen sus amiguitos, juegan con todo lo que encuentran a su mano, y tienen mascotas, son felices, y tienen sus campos donde ir, donde ellos quieren, Su espacio, gritan, corren, ;que no hacen!" (Abuelo).

\section{DISCUSIÓN Y CONCLUSIONES}

Los resultados muestran un concepto de salud y bienestar infantil inserto en la idea de equilibrio entre todos los seres del universo mapuche, este se traduce como küme mongen, küme felen (estar bien, en armonía con todo). Según 
Marileo $^{(21)}$, existe una concepción de salud relacionada al bienestar y al equilibrio del ser mapuche con todos los elementos que pueblan la naturaleza, en donde el ser humano es parte y está unido a ella. El mismo autor señala que todos los elementos son portadores de espíritu; el ser humano es un todo inseparable $\mathrm{y}$ es inconcebible dividir entre cuerpo y mente, o persona y naturaleza.

Por otra parte, para mantener la salud también hay que respetar las reglas de la convivencia entre ser y naturaleza, por ello a los niños se les enseña desde muy pequeños a ser bien comportados y respetuosos con las personas, animales y naturaleza ${ }^{(22) .}$ La salud de la infancia se asocia a su vez a un conjunto de valores que los niños/as deben demostrar desde pequeños, así, el cuidado infantil tiene componentes valóricos y éticos que apuntan a ser una persona buena, correcta, fuerte física $\mathrm{y}$ espiritualmente, $\mathrm{y}$ conocedora de sus raíces y su cultura ${ }^{(23)}$.

En consecuencia, el concepto de bienestar y salud en la infancia mapuche, se encuentra en la intersección de: valores culturales que apuntan a la formación de un ser persona; una epistemología del saber que se trasmite a los niños y niñas; una filosofía respecto del ser y la naturaleza; y un sistema de regulación del equilibrio entre dichos componentes. Por tanto, el concepto de salud occidental como "un estado de completo bienestar físico, mental y social y no solamente a la ausencia de enfermedades" (Organización Mundial de la Salud, 2020), es aún insuficiente para comprender la salud de la infancia mapuche, y menos culturalmente pertinente para evaluar la salud y desarrollo de estos ${ }^{(30)}$.
Dado el marco referencial de la relación naturaleza y la cultura, se indica que ser niño o niña mapuche sano, es crecer conforme al entorno ecológico y social, como una planta que se alimenta naturalmente, los niños/as tienen ganas de nutrirse, no tienen enfermedades y sus cuerpos son firmes y fuertes. Demuestran la adquisición de destrezas especialmente autonomía, son respetuosos, activos, vivaces, cariñosos, atentos a mirar y escuchar lo que ocurre a su alrededor e interesados en aprender. Todas estas habilidades o características, al parecer comunes a muchos niños, no son un listado general $o$ un enunciado que pueda desprenderse del análisis del contexto de la cultura mapuche. Niños y niñas, crecen en un entorno cultural cargado de simbolismo y sentido, el agradecimiento a un ser que nace como lo señalaba un padre por ejemplo, "cuando mi hijo nació en el hospital, yo lo recibí, le hablé mapudungun, le di la bienvenida a este mundo en mapudungun, ngiñatuke por él para que estuviera sano, que fuera un hombre fuerte, le pedí a chau ngenechen que él pudiera ser y cumplir con lo que nuestra gente antigua nos decía (...), ahora él tiene una edad y ha demostrado que él tiene esas cualidades". Estos son aspectos rituales y simbólicos que no se valoran al evaluar el apego de la familia a los/as hijos/as, por ejemplo. El crecer entre dos idiomas debe ser apreciado como una ventaja para el desarrollo intelectual de los niños mapuche, más que un problema en salud. Asimismo, vivir en un espacio familiar intergeneracional, que enseña roles, costumbres, y diversos conocimientos de la vida, es un factor de protección y una verdadera escuela para la infancia. Pensar 
Alarcón Muñoz AM, Garcés Zúñiga RE, Nahuelcheo Saldaña Y, Castro Garrido M

que niños o niñas pueden ser también llamados a cumplir roles culturales tradicionales es apreciarlos es en su identidad mapuche y a través de ellos a su propio pueblo ${ }^{(31)}$.

Los hallazgos de este estudio, demuestran que la salud y enfermedad son conceptos culturalmente definidos, los cuales dependen de un conjunto de valores y conocimientos que no siempre son congruentes con aquellos provenientes de los sistemas biomédicos de salud. Por lo tanto, el primer paso para trabajar con pueblos culturalmente diversos es comprender conceptos vitales de su existencia, de tal forma que la acción terapéutica o de acompañamiento en salud tenga efectividad y sentido.

Las formas culturales de criar, enseñar y aprender han sido fuente de estudio, desde hace varios años especialmente como una forma de hacer comprender la necesidad un enfoque psicoetnocultural que aborde la agencia infantil del propio niño-niña (procesos individuales), en función de la información colectiva social, familiar comunitario (procesos colectivos), y en un marco de definiciones culturales complejas (procesos culturales) ${ }^{(32,33}$ ).

Varios de los resultados aquí descritos, son coincidentes con hallazgos en estudios culturales de la infancia, especialmente aquellos sobre el vínculo del niño con la familia y su comu$\operatorname{nidad}^{(34,35)}$. Respecto de la importancia de la autonomía, los estudios de Murray ${ }^{(36)}$, describen cómo el ejercicio del control de la voluntad es intrínseco a la noción de persona y autonomía, y constituye una de las prácticas de socialización de niños mapuche a edad temprana. Desde una perspectiva social y política, el valor de la autonomía, como la posibilidad de construir un camino propio es de gran importancia en sociedades que sufren el peso de relaciones hegemónicas de poder. A los niños se les enseña a valerse por sí mismos, pero no en un sentido individualista, sino como la capacidad de levantarse a sí mismos ante la adversidad. el ejercicio de la propia voluntad y la construcción de un destino ${ }^{(37)}$.

Este estudio muestra una parte del proceso de cuidado y protección de la infancia mapuche que se desarrolla fundamentalmente en el ámbito de comunidades rurales. En el futuro se espera abordar los procesos de salud y desarrollo en familias urbanas, migrantes forzadas a espacios ajenos, donde el despliegue de la cultura propia puede ser un desafío para la familia mapuche.

Finalmente, es necesario señalar que, todos los/as niños/as deben crecer en un ambiente de paz y armonía como un contexto natural que otorga protección a la infancia ${ }^{(38)}$. No obstante, hay que alertar que muchos niños mapuche, viven hoy en día en territorios de conflicto armado, lo cual genera inseguridad e incertidumbre acerca del curso de su salud, bienestar y desarrollo futuro.

\section{AGRADECIMIENTOS}

Proyecto FONDECYT regular $\mathrm{n}^{\circ} 1150833$, Hitos y patrones intraculturales marcadores del desarrollo de niños mapuche de 0 a 4 años: contribuciones a la vigilancia del curso de la infancia indígena Universidad de La Frontera -TemucoChile.

Los autores declaran no tener conflictos de interés. 
El concepto cultural de bienestar en niñas y niños mapuche...

\section{REFERENCIAS BIBLIOGRÁFICAS}

1. Herrero Jaén Sara. Formalización del concepto de salud a través de la lógica: impacto del lenguaje formal en las ciencias de la salud. Ene. [Internet]. 2016 Ago [citado 2021 Jul 31];10(2).Disponible en: http://scielo.isciii.es/scielo.php?script= sci_arttext\&pid=S1988348X2016000200006\&lng=e.

2. Alfaro J, Casas F, López V. Bienestar en la infancia y adolescencia. Psicoperspectivas. [Internet]. 2015 [citado 2021 jul 31]; 14 (1). Disponible en:

https://scielo.conicyt.cl/scielo.php?scri $\mathrm{pt}=\mathrm{sci} \_$arttext\&pid=S07186924201500010000

3. Alarcón Ana M, Castro M, Astudillo P, Nahuelcheo Y. La paradoja entre cultura y realidad: El esfuerzo de criar niños y niñas mapuche en comunidades indígenas de Chile. Chungará [Internet]. 2018 dic [citado $2021 \mathrm{jul}$ 31]; 50 (4). Disponible en: https://dx.doi.org/10.4067/S071773562018005001601

4. Quidel J, Pichinao J. Haciendo crecer personas pequeñas en el Pueblo Mapuche. Temuco. Secretaria Regional de Educación de La Araucanía. Informe técnico; 2002.

5. Comisión interamericana de derechos humanos. Informe sobre la violencia institucional hacia la niñez mapuche en Chile. Washignton USA; 2012. Recuperado de: https://www.indh.cl/wpcontent/uploads/2012/08/informeviolencia-institucional-ninez-mapuche2012.pdf
6. Paillalef J. Los mapuches y el proceso que los convirtió en indios: psicología de la discriminación. Universidad Tecnológica Metropolitana ediciones: 2012.

7. Organización Mundial de la Salud. Centro de prensa. Notas descriptivas: La salud de los pueblos indígenas. Washington USA; 2017. Recuperado de:

http://www.who.int/mediacentre/factsh eets/fs326/es/

8. Domínguez A. (2017). Determinantes sociales de la salud en primera infancia indígena en Chile [Tesis para optar al grado de maestría Salud Pública]. Santiago: Universidad de Chile; 2017.

9. PAHO, WHO. La salud de los pueblos indigenas. 2007; Disponible en: paho.org/hq/index.php?option=com_c ontent $\&$ view=article $\&$ id=1475:saludpueblos-indigenas-atencion-primariainterculturalidad\&Itemid=820\&lang=e $\mathrm{s}$

10. Díaz A, Vargas R. Situación de salud y nutrición de niños indígenas y niños no indígenas de la Amazonia peruana. Revista panamericana de salud pública [Internet]. 2009;38:49-56. Disponible en:

https://www.scielosp.org/article/rpsp/2 015.v38n1/49-56

11. Amigo H, Bustos P. Salud y nutrición del niño indígena chileno. Saude pu [Internet]. 2019;35. Disponible en: https://www.scielo.br/j/csp/a/rqm33Td StqVbSbSNRRCC77r/?lang=es\&form at=pdf

12. Instituto Nacional de Estadísticas. Censo de población 2017. Disponible en:

https://www.censo2017.cl/descargas/h 
Alarcón Muñoz AM, Garcés Zúñiga RE, Nahuelcheo Saldaña Y, Castro Garrido M

ome/sintesis-de-resultados-

censo2017.pdf

13. Ministerio de desarrollo social. CASEN 2017 [Internet]. 2017. Disponible en: http://observatorio.ministeriodesarrollo social.gob.cl/casenmultidimensional/casen/docs/Resultad os_nna_casen_2017.pdf.

14. BANCO MUNDIAL, MINSAL. Plan para Pueblos Indígenas. Santiago de Chile [Internet]. 2017. Disponible en: https://www.minsal.cl/wpcontent/uploads/2017/04/PPI-

_Chile_HESSP-7-de-abril-2017MINSAL-OK.pdf

15. Escobar B, Paravic-Klijn T. La transculturalidad, elemento esencial para mejorar la atención en salud y de enfermería. Enfermería actual en Costa Rica. [Internet] 2017 [citado $20121 \mathrm{Jul}$ 31]; 32 (1). Disponible en: https://www.scielo.sa.cr/pdf/enfermeri a/n33/1409-4568-enfermeria-3300073.pdf

16. Ribeiro L, Silva J. Diversidad cultural e implicaciones en el entorno social. En: Leal Filho We, Azul AM, Brandli L., Lange Salvia A., Özuyar PG, Wall T. editores. Reduced Inequalities. Enciclopedia de los Objetivos de Desarrollo Sostenible de la ONU. Springer Cham USA; 2021.

17. Eipperle M. Aplicación de los tres modos de decisión y actuación en la práctica avanzada de atención primaria. En: McFarland MR, Wehbe-Alamah HB editores. La cultura de Leininger cuida la diversidad y la universalidad: una teoría de enfermería en todo el mundo. 3a ed. Burlington: Jones y Bartlett Learning USA; 2018. p.317344.
18. Capano A, Ubach A. Parenting styles, positive parenting and parents formation. Ciencias Psicológicas [Internet]. 2013 [citado 2021 Jul 31]; 7(1). Disponible en: http://www.scielo.edu.uy/scielo.php?sc ript=sci_arttext\&pid=S168842212013000100008\&lng=es\&tlng=es

19. Lancy D. The Anthropology of Childhood: Cherubs, Chatel, Changelins. Cambrigde: University Press, St. Ives; 2016.

20. Quilaqueo D, Quintriqueo S, Torres H. Características epistémicas de los métodos educativos Mapuche. Revista Electrónica de Investigación Educativa. [Internet] 2016 [citado $2021 \mathrm{Jul}$ 31]; 18 (1). Disponible en: http://www.scielo.org.mx/scielo.php?s cript=sci_arttext\&pid=S160740412016000100011\&lng=es\&tlng=es

21. Marileo A. El mundo Mapuche. En: Citarella L. editor. Medicinas y Culturas en La Araucanía. $2^{\mathrm{a}}$ ed. Santiago: Pehuen Chile; 2018 p.61-71.

22. Quilaqueo R, Quintriqueo M. Saberes educativos mapuches: un análisis desde la perspectiva de los kimche. Polis [Internet]. 2010 [citado 2021 Jul 31]; 9 (26). Disponible en: https://dx.doi.org/10.4067/S071865682010000200016.

23. Quidel J. El quiebre ontológico a partir del contacto mapuche hispano. Chungará [Internet]. 2016 [citado 2021 Jul 31]; 48(4). Disponible en: https://dx.doi.org/10.4067/S071773562016000400016

24. Meza-Calfunao E, Díaz-Fuentes R, Alarcón-Muñoz AM. ¿Qué es küme mogen mapuche? Concepto e implicancias en salud pública y 
El concepto cultural de bienestar en niñas y niños mapuche...

comunitaria. Salud Publica Mex [Internet]. 28 de junio de 2018 [citado 29 de julio de 2021];60(4, jul-ago):38021. Disponible en: https://saludpublica.mx/index.php/spm /article/view/8988.

25. Heckmann J. Giving kids a fair chance. Boston: review Books; 2012.

26. Castilla-Peón María Fernanda. Bienestar infantil: ¿es posible medirlo? Bol. Med. Hosp. Infant. Mex. [revista en la Internet]. 2014 Feb [citado 2021 Ago 01]; 71(1): Disponible en: http://www.scielo.org.mx/scielo.php?s cript=sci_arttext\&pid=S166511462014000100010\&lng=es

27. Bonilla-García M, López-Suarez A. Ejemplificación del proceso metodológico de la teoría fundamentada. Cinta de Moebio. [Internet] 2016 [citado 2021 Jul 31]; 57 ():305-15. Disponible en: https://www.scielo.cl/scielo.php?script =sci_isoref $\&$ pid $=$ S0717-

$554 X 2016000300006 \& \operatorname{lng}=e s \& \operatorname{lng}=\mathrm{e}$ $\mathrm{s}$

28. Charmaz K. Evolving grounded theory and social justice inquiry. En: Denzin $\mathrm{N}$, Lincoln $\mathrm{Y}$. editores. The sage handbook of qualitative research. 5a ed. California: SAGE USA; 2018. p. 41143.

29. Guba EG, Lincoln YS. Effective evaluation: improving the usefulness of evaluation results through responsive and naturalistic approaches. San Francisco: Jossey-Bass; 1981.

30. Hasen Narváez Felipe Nayip. Interculturalidad en salud: competencias en prácticas de salud con población indígena. Cienc. Enfe33rm. [Internet]. 2012 [citado 2021 Jul 29];
18(3): Disponible en: http://www.scielo.cl/scielo.php?script= sci_arttext\&pid=S071795532012000300003\&lng=es

31. Mariman J. Autodeterminación. Ideas Políticas Mapuche en el Albor del Siglo XXI. Pehuen; 2012.

32. Rogoff B, Aceves-Azuara I, Dayton A. Learning by Observing and Pitching In. En: K. Peppler editora, The Sage encyclopedia of out-of-school learning. Vol. 1. Cambridge: SAGE USA; 2017. p. 425-428.

33. Keller H. Universality claim of attachment theory: children's socioemotional development across culture. PNAS [Internet]. 2018 [citado 2021 Jul 30]; 115(45): 11414-11419. Recuperado de: https://www.pnas.org/content/115/45/1 1414.

34. Remorini, Carolina, Palermo, María Laura, "Los míos ... ¡todos asustados!". vulnerabilidad infantil y trayectorias de desarrollo en los valles Chalchaquíes salteños. Mitológicas [Internet]. 2016; XXXI (): 83-112. Recuperado de: https://www.redalyc.org/articulo.oa?id $=14649178005$.

35. Tassinari A, Guedes C. Al ritmo de la comunidad: enseñanza y aprendizaje entre niños indígenas GalibiMarworno. Anthropologica [Internet]. 2015 [citado 2021 Jul 31]; XXXIII (35):173-203.Recuperado de http://www.scielo.org.pe/scielo.php?pi $\mathrm{d}=\mathrm{S} 0254$ -

92122015000200008\&script=sci_abstr act

36. Murray M, Bowen S, Segura N, Verdugo Paiva MO. Aprehender el voluntariado en la socialización 
Alarcón Muñoz AM, Garcés Zúñiga RE, Nahuelcheo Saldaña Y, Castro Garrido M

temprana: crianza de "personitas" entre familias rurales mapuche. Ethos. 2015; 43 (4): 376-401.

37. Cocking R. Ecologically valid frameworks development accounting for continuities and discontinuities across contexts. En: Greenfield P, y Cocking R. editores. Cross-cultural roots of minority child development. 3a ed. New Yersey: Lawrence Erlbaum Associates USA; 2008. p. 393-409.

38. UNICEF. Desarrollo de la primera infancia. Fondo de las naciones unidas para la infancia. Nueva York. [Internet] 2017; Disponible en: https://www.unicef.org/es/desarrollode-la-primera-infancia 